\title{
Technical note: Validation of data loggers for recording lying behavior in dairy goats
}

\author{
G. Zobel, ${ }^{*}$ D. M. Weary, ${ }^{*}$ K. Leslie, $\dagger$ N. Chapinal, ${ }^{*}$ and M. A. G. von Keyserlingk ${ }^{* 1}$ \\ *Animal Welfare Program, Faculty of Land and Food Systems, University of British Columbia, 2357 Main Mall, Vancouver, British Columbia, \\ V6T 1Z4, Canada \\ †Department of Population Medicine, University of Guelph, 2536 Stewart Building, Guelph, ON, N1G 2W1 Canada
}

\section{ABSTRACT}

Changes in standing and lying behavior are frequently used in farm animals as indictors of comfort and health. In dairy goats, these behaviors have primarily been measured using labor-intensive video and live observation methodologies. The aim of this study was to validate accelerometer-based data loggers for use in goats. Two commercial dairy goat farms in Ontario were enrolled; goats were fitted with data loggers on their rear left legs and the pens were equipped with video. Data loggers compared well with video in identifying lying and standing events on both farms (farm 1 and 2 , respectively: sensitivity $=99.7$ and $99.8 \%$, specificity $=99.5$ and $99.4 \%$, false readings $=0.43$ and $0.36 \%$ ). The loggers were also able to record if the goat was lying on her left or right side (farm 1 only: sensitivity $=99.9 \%$, specificity $=99.3 \%$, false readings $=0.38 \%$ ), but these measures were only accurate if the loggers were attached with sufficient tension to prevent logger rotation. The mature does enrolled on farm 1 spent $14.5 \pm 1.0 \mathrm{~h} / \mathrm{d}$ lying down and frequently changed lying side even within a single lying bout $(24 \pm 5$ shifts/d between left and right sides and $16 \pm 5$ lying bouts/d). The young goats on the second farm averaged just 8.5 $\pm 3.2 \mathrm{~h} / \mathrm{d}$ in lying time, and spread this time over 8 \pm 4 bouts/d. Data loggers accurately measured lying time and lying bouts in mature does and younger goats on both farms, and lying laterality (e.g., left and right lying sides) in mature does on farm 1.

Key words: accelerometer, activity, does, welfare

\section{Technical Note}

Measures of standing and lying behavior have been used in the assessment of welfare of farm animals in many applications. For instance, in dairy cattle, links have been found between increased standing and mas-

Received July 17, 2014.

Accepted October 31, 2014.

${ }^{1}$ Corresponding author: nina@mail.ubc.ca titis (Medrano-Galarza et al., 2012), claw horn lesions (Proudfoot et al., 2010), overall lameness (Ito et al., 2010), and bedding, cleanliness, and flooring (Ito et al., 2014). Less work has been done on dairy goats, but studies to date have used changes in lying behavior to assess how goats respond to separation and reintegration within groups (Patt et al., 2013), to determine the effects of different stocking densities (Loretz et al., 2004; Andersen and Bøe, 2007), and to evaluate the positive effects of environmental enrichment (Aschwanden et al., 2009). In the majority of goat studies to date, video and direct observation were used to assess lying and standing time and bouts. Unfortunately, video can be difficult to install in some commercial farms and video recordings are labor intensive to analyze. Live observations may disturb the goats' behavior (due to presence of the observer) and, again, are labor intensive, especially if many animals are followed over an extended period. Data loggers can automate recordings and provide more detailed coverage over a long period than is typically practical for video or live observations. Although one study has utilized data loggers (different from those being validated in this study), it was not indicated whether these loggers were validated for use in goats (Patt et al., 2012).

Data loggers, specifically accelerometers (e.g., devices that record $g$-force acceleration values), have been readily adopted into research. Several devices are commercially available for use on dairy cows. For instance, the IceTag Sensor (IceRobotics Ltd., Edinburgh, UK) has been successfully used to monitor lying behavior (Gibbons et al., 2012), but the size of these products makes them unsuitable for use on goats. The Hobo Pendant $\mathrm{G}$ data logger (Onset Computer Corporation, Bourne, MA) is smaller and can be used on goats, but to date this device has only been validated for use on dairy cows (Ledgerwood et al., 2010) and dairy calves (Bonk et al., 2013). The aim of the current study was to validate the use of these data loggers for goats.

Our 3 objectives were to (1) determine appropriate cut-off points for coding lying and standing, as well as lying side, in the raw $g$-force data collected by Hobo 
data loggers; (2) determine a suitable correction factor for dealing with data abnormalities caused by other activities (e.g., perching, scratching, and urination); and (3) assess the validity of using the data loggers on both mature, pregnant does, as well as younger, nonmilking goats.

Two commercial farms in southern Ontario, Canada, were enrolled. The Hobo Pendant G data loggers were attached vertically (with the rounded base closest to the ground), on the inside of the rear left leg (above the metatarsophalangeal joint) of a subset of goats on each farm. This orientation resulted in the data logger's xaxis pointing upwards and the z-axis pointing inwards toward the animal's leg, with the logger label showing. Attachment was done with veterinary self-adherent bandage (Vetrap, 3M, St. Paul, MN) and pieces of foam to minimize any chafing. Loggers were set to record in 1-min intervals; this allows for more than 3 wk of continuous data collection. Due to the vertical placement of the logger, only the $g$-forces for the $\mathrm{x}$ - and z-axes were recorded (the $y$-axis does not change when the logger is vertically oriented). Logger data were downloaded via HoboWare Pro V3 (Onset Computer Corporation) and raw $g$-force values were exported as CSV files, which were then imported into SAS (SAS Institute Inc., Cary, $\mathrm{NC})$ for further analyses.

On farm 1, 4 mature, multiparous Saanen $\times$ Alpine crossbred does were monitored using video. Prior to monitoring, these does were housed in a group with 30 other late gestation does (approximately 120-130 d); all does were fitted with data loggers. The video-monitored does were selected based on differences in color and ear characteristics (e.g., black, white, gray, and La Mancha). During the monitoring period, these 4 does were moved to a smaller pen $(3 \times 4 \mathrm{~m})$ immediately adjacent to the home pen. All management remained the same as in the home pen. All goats had ad libitum access to water and a chopped hay and silage mixture. Approximately $100 \mathrm{~g}$ of corn and commercial supplement per goat was top dressed 3 times daily. Does were not milking at the time of observation. Two cameras were placed above the monitored pen (Panasonic HDC TM-900, Taiwan) and recorded video continuously for $3 \mathrm{~d}$. All instances of lying and standing behavior were coded from the $72 \mathrm{~h}$ of video (Observer XT, Noldus Information Technology, Leesburg, VA). Additionally, all other behaviors with potential to affect the data logger orientation (e.g., perching on back legs, urination, and lifting rear legs for scratching) were noted. For comparison to logger data, daily values (for each goat on each of the $3 \mathrm{~d}$ ) were calculated for lying time, lying bouts, as well as shifts in lying position between the left and the right sides. Standing behavior (i.e., time, bouts, bout duration) was calculated for descriptive statistics only. One 3-h block of video was rewatched to determine intraobserver reliability for distinguishing between lying and standing events (Cohen's kappa, $\kappa=$ 0.84). Lying and standing behaviors were also measured using loggers (but not video) for the 30 does remaining in the home pen; these results from the home pen does are provided for descriptive purposes only.

On farm 2, 5 Saanen crossbred doelings (nonbred) and 1 Saanen crossbred wether (neutered male) were housed in a single $3-\times 6$-m pen bedded with straw. All goats were between 8 and 12 mo old. These animals were approximately half the size of the mature, late gestation does on farm 1. All 6 animals had data loggers attached as described above. Individual animals were marked with hair dye to facilitate identification on video. Hay was fed twice a day and ad libitum water access was provided. One camera (Panasonic HDC TM-900) recorded video continuously for $3 \mathrm{~d}$. Lying and standing behavior was continuously coded from the video. The full $72 \mathrm{~h}$ of video was used to calculate 3 -d values for lying time and lying bouts for each goat. Differences in lying side (between left and right), as well as perching, scratching, and urination behaviors, could not be reliably coded on this farm due to the camera's view angle (constrained by a low ceiling). One 3 -h block of video was rewatched to determine intraobserver reliability for discerning between lying and standing events (Cohen's kappa, $\kappa=0.88$ ).

\section{Objective 1}

The raw data on the $\mathrm{x}$ - and $\mathrm{z}$-axes ranged from -3.2 to $3.15 \mathrm{~g}$. To avoid any data-handling issues caused by negative data points, a value of 3.2 was first added to the raw data collected by the data loggers (Ledgerwood et al., 2010), resulting in an adjusted range of 0 to 6.35. To determine appropriate cut-off points, PROC UNIVARIATE (SAS 9.2) was used to generate separate frequency distributions of the adjusted $\mathrm{x}$ - and $\mathrm{z}$-axis values for the data from farm 1 . Cut-off points established by Ledgerwood et al. (2010) for dairy cattle were first plotted onto these distributions and used to assist in the visual identification of 6 potentially feasible cutoff points for use in goats. Next, the adjusted $x$ - and z- axis values were converted to binary values, using the potential cut-off points to associate 1 for standing and 0 for lying (x-axis) and 1 for lying on the right side and 0 for lying on the left side (z-axis). This created a 3-d, minute-by-minute data set for each goat, which were then combined into data sets for each axis. A rotated logger resulted in the z-axis data of 1 goat being discarded from the data for the group of does monitored by video. Video data was also converted into a minute-by-minute binary data set and merged with 
the data sets from the data loggers. The final data sets used in the video comparison were comprised of 17,280 data points (x-axis) and 12,960 data points (z-axis). PROC FREQ (SAS 9.2) was used to determine sensitivity, specificity, and percentage of false readings (i.e., when the data logger coded lying as standing and vice versa) for each possible cut-off point (Table 1). The best cut-off points were chosen based on the highest sum of sensitivity and specificity, as well as the lowest false readings; these cut-off points were determined to be 2.75 (x-axis) and 3.75 (z-axis). Whereas the standing or lying cut-off point was similar to previously established values in dairy cows (x-axis: 2.55; Ledgerwood et al., 2010), the cut-off point for determining left and right lying side was different (z-axis: 3.025; Ledgerwood et al., 2010). The reason for this discrepancy is not clear, but may be due to a combination of factors including differences in anatomy, body size, lying posture, and bedding. The established cut-off points are suitable for use when the loggers are oriented with the $\mathrm{x}$-axis pointed upwards and the z-axis pointing inward. If loggers are applied upside down or backward, the raw data must be inversed before the adjustment value of 3.2 (discussed previously) is added.

\section{Objective 2}

Perching on rear legs, lifting rear legs to scratch, and urination can alter the orientation of the rear legs and potentially result in an apparent transition from standing to lying or vice versa. As these behaviors often occur for less than $1 \mathrm{~min}$, the 1-min data-logging interval avoided capturing many of these erroneous data points. Nonetheless, as video analysis revealed that farm 1 goats spent an average of $19 \pm 12$ min daily perching on rear legs, lifting rear legs to scratch, or urinating, it is pertinent to understand the potential effect of these behaviors on the loggers. Therefore, several filters were assessed. The raw data from each axis (with a value of 3.2 added to ensure all positive numbers) was first converted to binary values using the established cut-off points from objective 1, then filters were applied to either remove single (1-min event) or double (2-min event) recordings of zeroes in a string of ones or vice versa. Filtered data were then summarized to determine daily lying time and bouts (x-axis) and shifts in lying position between left and right sides ( $\mathrm{z}$ axis) for each goat for each filter. Standing behaviors (i.e., time, bouts, bout duration) were also calculated for descriptive statistics only. Next, Pearson correlation coefficients (PROC CORR; SAS 9.2) between video and data logger lying data were used to assess filters. A total of 12 data points ( $3 \mathrm{~d}$ for 4 goats) were used for the $\mathrm{x}$-axis data and 9 data points $(3 \mathrm{~d}$ for 3 goats, due to a rotated logger) were used for the z-axis data. Use of a 1-min event filter resulted in the best relationships between video and data logger lying data estimates (Table 2).

Table 1. Sensitivity, specificity, and false readings between video data and data logger data used to establish cut-off points for the $\mathrm{x}$ - and $\mathrm{z}$ - axis (farm 1, video-monitored does)

\begin{tabular}{lccc}
\hline Cut-off point $^{1}$ & $\begin{array}{c}\text { Sensitivity, } \% \\
(95 \% \mathrm{CI})\end{array}$ & $\begin{array}{c}\text { Specificity, } \% \\
(95 \% \mathrm{CI})\end{array}$ & $\begin{array}{c}\text { False } \\
\text { readings, } \%\end{array}$ \\
\hline X-axis (lying/standing) $^{2,3}$ & & & \\
2.45 & $99.1(98.9-99.3)$ & $98.7(98.4-98.9)$ & 1.2 \\
2.55 & $99.1(98.9-99.3)$ & $98.8(98.6-99.0)$ & 1.1 \\
2.65 & $99.1(98.9-99.3)$ & $98.9(98.7-99.1)$ & 1.0 \\
2.75 & $99.1(98.8-99.3)$ & $99.0(98.8-99.2)$ & 0.98 \\
2.85 & $99.0(98.7-99.2)$ & $99.0(98.9-99.3)$ & 0.95 \\
2.95 & $98.8(98.5-99.0)$ & $99.1(98.9-99.3)$ & 1.0 \\
Z-axis (left/right) & & & \\
3.025 & $61.9(60.1-63.1)$ & $99.7(99.1-99.9)$ & 33.4 \\
3.55 & $99.0(98.6-99.3)$ & $99.8(99.6-99.9)$ & 0.65 \\
3.65 & $99.8(99.6-99.9)$ & $99.8(99.6-99.9)$ & 0.34 \\
3.75 & $99.9(99.7-100.0)$ & $99.4(99.1-99.7)$ & 0.22 \\
3.85 & $99.9(99.7-100.0)$ & $97.8(97.3-98.3)$ & 1.1 \\
3.95 & $99.9(99.7-100.0)$ & $93.2(92.4-94.0)$ & 3.4 \\
\hline
\end{tabular}

${ }^{1}$ All raw $g$-force data were first adjusted by 3.2 to ensure all values were positive.

${ }^{2}$ Possible test cut-off points were chosen around previously established cut-off point (dairy cows $=2.55$; Ledgerwood et al., 2010).

${ }^{3}$ Total data points $=17,280$ (4 goats, $3 \mathrm{~d}, 1,440$ data points per day).

${ }^{4}$ We found poor sensitivity when using the previously established cut-off point (dairy cows $=3.025$; Ledgerwood et al., 2010) and therefore increased the starting test cut-off point to 3.55 as identified visually from the frequency distribution of the raw z-axis data.

${ }^{5}$ Due to a rotated logger, the z-axis data of 1 goat was discarded, resulting in total data points $=12,960(3$ goats, $3 \mathrm{~d}, 1,440$ data points per day). 
Table 2. Pearson correlation coefficients between daily lying behaviors recorded from video and from data loggers (farm 1, video-monitored does)

\begin{tabular}{|c|c|c|c|}
\hline Behavior & No filter & 1 -min filter ${ }^{1}$ & 2 -min filter ${ }^{2}$ \\
\hline \multicolumn{4}{|l|}{ Lying (x-axis) $)^{3}$} \\
\hline Time & $1.0 * * *$ & $1.0^{* * *}$ & $1.0 * * *$ \\
\hline Bouts & $0.65^{*}$ & $1.0 * * *$ & $0.92^{* * *}$ \\
\hline \multicolumn{4}{|l|}{ Lying side (z-axis) ${ }^{4}$} \\
\hline Side changes & $0.85^{* *}$ & $0.99^{* * *}$ & $0.99 * * *$ \\
\hline Right side time & $1.0 * * *$ & $1.0 * * *$ & $1.0^{* * *}$ \\
\hline Right side bouts & $0.69^{*}$ & $0.98^{* * *}$ & $0.98 * * *$ \\
\hline Left side time & $1.0 * * *$ & $1.0^{* * *}$ & $1.0 * * *$ \\
\hline Left side bouts & $0.93^{* *}$ & $0.99 * * *$ & $0.93^{* *}$ \\
\hline
\end{tabular}

${ }^{1}$ Logger data filtered to remove single observations (1-min event).

${ }^{2}$ Logger data filtered to remove double observations (2-min event).

${ }^{3}$ Daily behaviors for $3 \mathrm{~d}$ of data for 4 goats $(\mathrm{n}=12)$.

${ }^{4}$ Due to a rotated logger, the $\mathrm{z}$-axis data from one goat was discarded, resulting in $3 \mathrm{~d}$ of data for 3 goats $(\mathrm{n}=9)$.

${ }^{*} P<0.05,{ }^{* *} P<0.01,{ }^{* * *} P<0.0001$.

The data filters were also assessed using plots of the difference between the 2 measurement methodologies against their mean, following Bland and Altman (1986; Figure 1). For lying time, the lowest mean difference between video and data loggers $(-2.3 \pm 3.0 \mathrm{~min})$ was found with the 1-min filter. Whereas a mean difference of 0 min would be ideal, the relative flexibility of goats in their leg position while lying meant that on a few occasions some data points were very close to the cut-off point; this, in turn, resulted in a few lying points (often at the end of a lying bout when a goat was rising and stretching) being recorded incorrectly by the logger. Using either no filter or the 2-min filter resulted in larger mean differences $(-4.3 \pm 3.5$ and $-4.6 \pm 3.4 \mathrm{~min}$, respectively). Similarly, the 1-min filter also resulted in the lowest mean difference between video and data loggers for lying side changes $(0.2 \pm 0.8$ changes). As anticipated, applying no filter resulted in larger negative mean difference between video and data loggers for lying side changes $(-7.9 \pm 2.7$ changes $)$. Applying the 1-min filter allowed for removal of some perching, scratching, and urination events that the data logger would otherwise incorrectly code as a lying event. Because goats are relatively active, with numerous shifts between standing and lying and between the left and right side, applying the 2-min filter resulted in the data loggers underestimating the number of lying side changes (mean difference: $0.8 \pm 1.1$ changes).

Other measures followed a similar pattern; all benefited most from the 1-min filter (lying bouts: $0.5 \pm$ 0.8 ; left side lying time: $1.0 \pm 5.9$; left side bouts: 0.1 \pm 0.3 ; right side lying time: $-3.4 \pm 4.5$; right side lying bouts: $0.1 \pm 0.8$ ). For right and left lying time, we observed 1 outlier that the logger coded as $12 \mathrm{~min}$ on the right side lying but video showed that the goat was lying on her left side (plots not shown). This was the only such outlier on the Bland-Altman plots and video analysis showed that the goat was lying in an atypical position (with legs outstretched behind her).

The complete minute-by-minute data set, filtered using the 1-min filter (PROC FREQ; SAS, 9.2), was utilized to determine sensitivity, specificity, and percentage of false readings between x-axis standing (1) and lying (0), as well as z-axis left side lying (1) and right side lying (0), from the video versus the data loggers. Data loggers showed high sensitivity and specificity and low false readings ( $\mathrm{x}$-axis: sensitivity $=99.7 \%$, specificity $=99.5 \%$, false readings $=0.43 \%$; z-axis: sensitivity $=99.9 \%$, specificity $=99.3 \%$, false readings $=0.38 \%$ ).

Table 3 shows descriptive statistics for all the measured lying and standing behaviors for the 4 videomonitored does, as well as the 30 does which remained in the adjacent home pen from which the 4 does were chosen. Higher lying times $(14.5 \pm 1.0 \mathrm{~h} / \mathrm{d}$ for videomonitored does; $14.5 \pm 1.4 \mathrm{~h} / \mathrm{d}$ for home pen does) were found compared with previous work with goats [e.g., $12 \mathrm{~h} / \mathrm{d}$ in Patt et al. (2013) from direct observation] and work with dairy cows [e.g., $11-12 \mathrm{~h} / \mathrm{d}$ in Ito et al. (2009) from loggers]. The difference may be explained by stage of gestation of the goats; the goats were in late gestation and therefore likely motivated to spend more time lying down.

Lying bouts and lying bout duration in dairy cows are typically calculated under the assumption that at least 1 min of standing occurs between each lying bout (allowing for the total number of lying bouts to be a sum of left and right side lying bouts). This approach is justified, as these animals generally have relatively long lying bout durations and standing events intersecting changes in lying sides. However, video from the current study showed that dairy goats differed from cows in the way they shifted between lying bouts and left and right lying sides, perhaps due to a difference in housing as well as size compared with dairy cattle. Goats frequently shifted between left and right lying sides within the same lying bout, and they often did this very quickly (on average just $8 \pm 5 \mathrm{~s}$ ) by rising on their rear legs and dropping to the opposite side. Shifts in lying sides were also associated with brief $(41 \pm 12 \mathrm{~s})$ standing events triggered by urination and defecation. Both of these types of events averaged less than $1 \mathrm{~min}$ and thus would often be missed by data loggers set to record every minute. Rather than having a standing bout between each side change, as is typically seen in cows, the logger data in goats would shift abruptly from one side to another. Therefore, methods used for calculating lying bouts in dairy cows (i.e., sum of left and right lying bouts) would overestimate the actual number of lying bouts in goats. We calculated lying bouts according to 

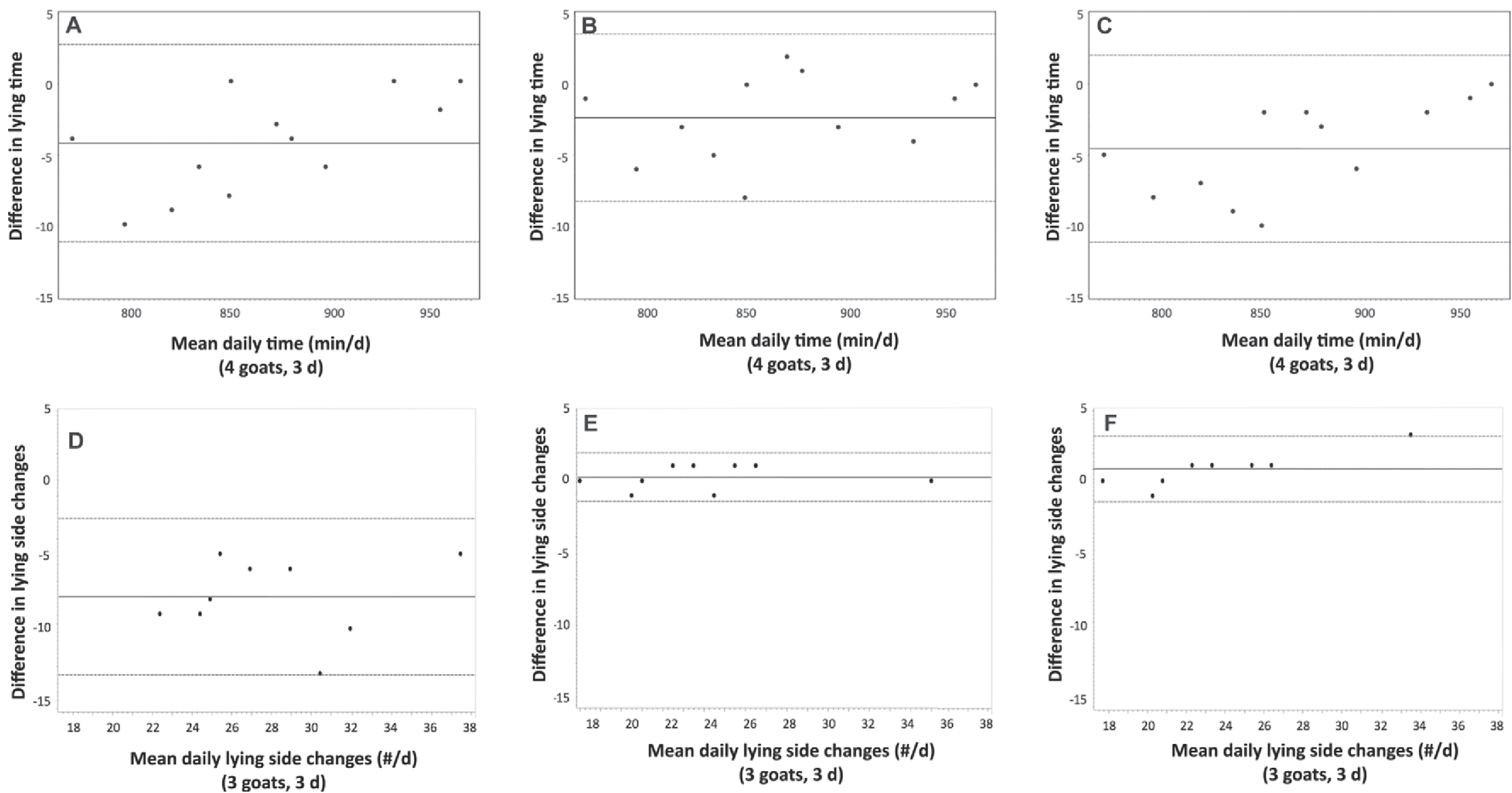

Figure 1. Bland-Altman plots comparing the difference in means (video-data logger) for lying time (A, B, C) and lying side changes (D, E, F), using no filter (A, D), a 1-min event filter (B, E), and a 2-min event filter $(\mathrm{C}, \mathrm{F})$. A solid line indicates mean difference and dotted lines represent 2 SD from the mean difference. The $\mathrm{x}$-axis shows the average of video and data logger values. Lying time (x-axis) data are for $3 \mathrm{~d}$ from 4 goats $(\mathrm{n}=12)$ and lying side changes $(\mathrm{z}$-axis) are for $3 \mathrm{~d}$ from 3 goats $(\mathrm{n}=9)$ due to a rotated logger on 1 goat. (farm 1, video-monitored does). 
Table 3. Descriptive statistics for daily lying and standing behaviors, using $3 \mathrm{~d}$ of data per goat, for both the does monitored by video and the remaining goats from which these monitored does were chosen (farm 1)

\begin{tabular}{|c|c|c|c|c|c|c|}
\hline \multirow[b]{2}{*}{ Behavior } & \multicolumn{3}{|c|}{ Video-monitored does ${ }^{1}$} & \multicolumn{3}{|c|}{ Home pen does ${ }^{2}$} \\
\hline & Mean & $\mathrm{SD}$ & Range & Mean & SD & Range \\
\hline \multicolumn{7}{|l|}{ Lying/standing (x-axis) } \\
\hline Lying time (min) & 870 & 61 & $771-965$ & 868 & 83 & $654-1,099$ \\
\hline Lying bout duration (min/bout) & 62 & 20 & $43-110$ & 73 & 19 & 41-135 \\
\hline Standing time $(\min )$ & 571 & 61 & $475-669$ & 572 & 83 & $341-786$ \\
\hline Standing bout duration ( $\mathrm{min} /$ bout) & 40 & 15 & $22-64$ & 53 & 20 & $20-126$ \\
\hline Lying/standing bouts (n) & 15 & 5 & $8-22$ & 12 & 4 & $5-22$ \\
\hline \multicolumn{7}{|l|}{ Laterality (z-axis) } \\
\hline Lying side changes $(\mathrm{n})$ & 24 & 5 & $18-35$ & 21 & 5 & $10-34$ \\
\hline Right side lying time ( $\min$ ) & 399 & 100 & $264-523$ & 432 & 117 & $143-921$ \\
\hline Right side lying bouts (n) & 11 & 4 & 7-19 & 11 & 3 & 3-18 \\
\hline Left side lying time (min) & 468 & 89 & $347-616$ & 440 & 113 & $670-42$ \\
\hline Left side lying bouts (n) & 13 & 2 & $10-16$ & 11 & 3 & $3-20$ \\
\hline
\end{tabular}

${ }^{1}$ Data for $3 \mathrm{~d}$ of behavior of the 4 video-monitored does $(\mathrm{n}=12$ for lying/standing, $\mathrm{x}$-axis; due to 1 rotated logger, $\mathrm{n}=9$ for laterality, z-axis).

${ }^{2}$ Data for $3 \mathrm{~d}$ of behavior of 30 does which remained in the home pen from which video-monitored does were chosen $[\mathrm{n}=90$ for lying/standing (x-axis); due to 6 rotated loggers, $\mathrm{n}=72$ for laterality, $\mathrm{z}$-axis $]$.

the $\mathrm{x}$-axis data (e.g., changes between lying and standing events), and the sum of shifts between right and left lying sides (recorded on the z-axis) was referred to as lying side changes.
Lying bout duration (i.e., daily lying time/daily number of lying bouts) has been used as a possible indicator of potential discomfort in cattle (Gomez and Cook, 2010; Ito et al. 2014), but we suggest caution

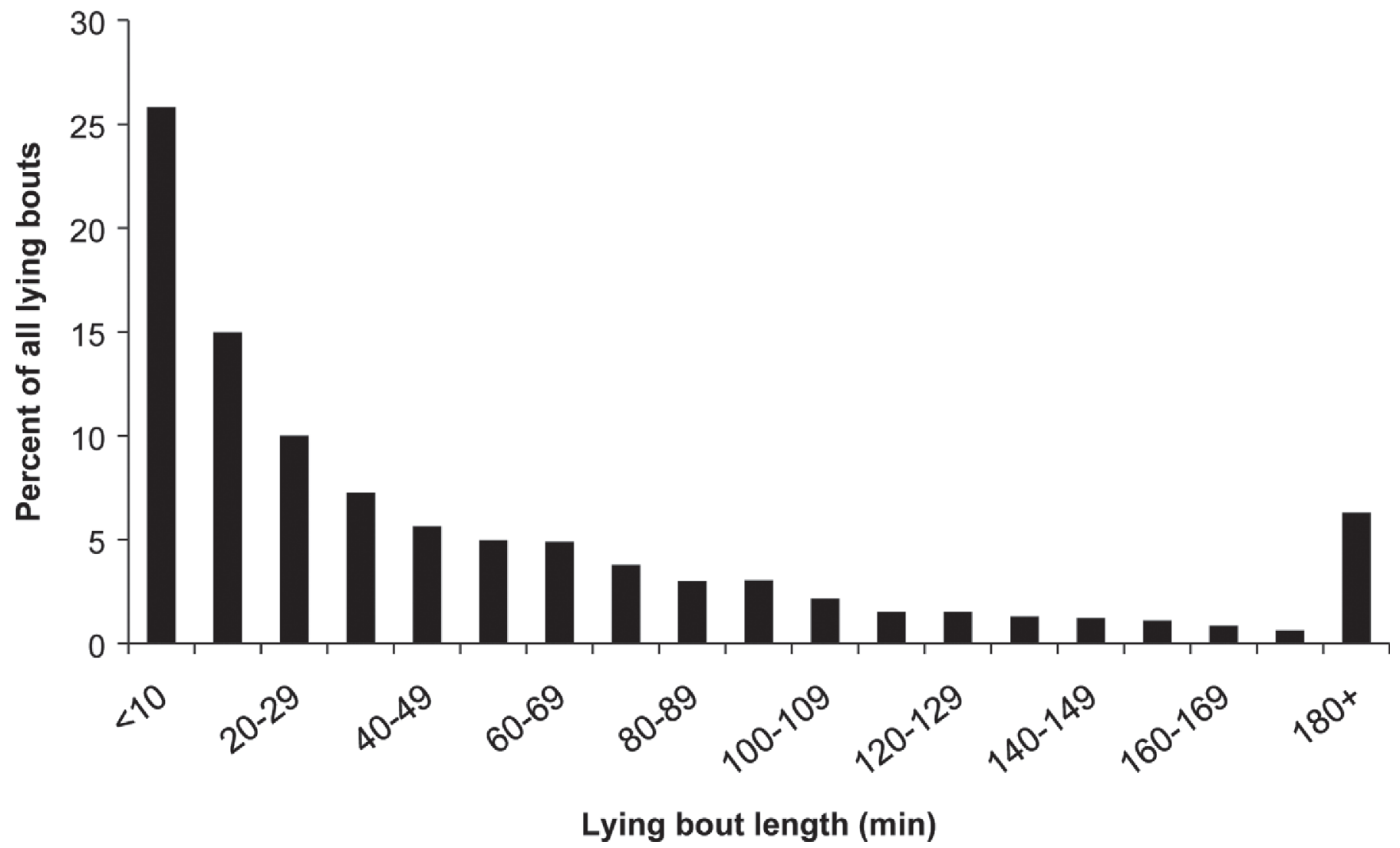

Figure 2. Frequency distribution of lying bout durations for 34 adult dairy goats over $3 \mathrm{~d}$ (farm 1, video-monitored does and home pen does), plotted in 10-min bins. Does were extremely active and split their daily lying times into many short lying bouts (e.g., less than 10 min in length). 


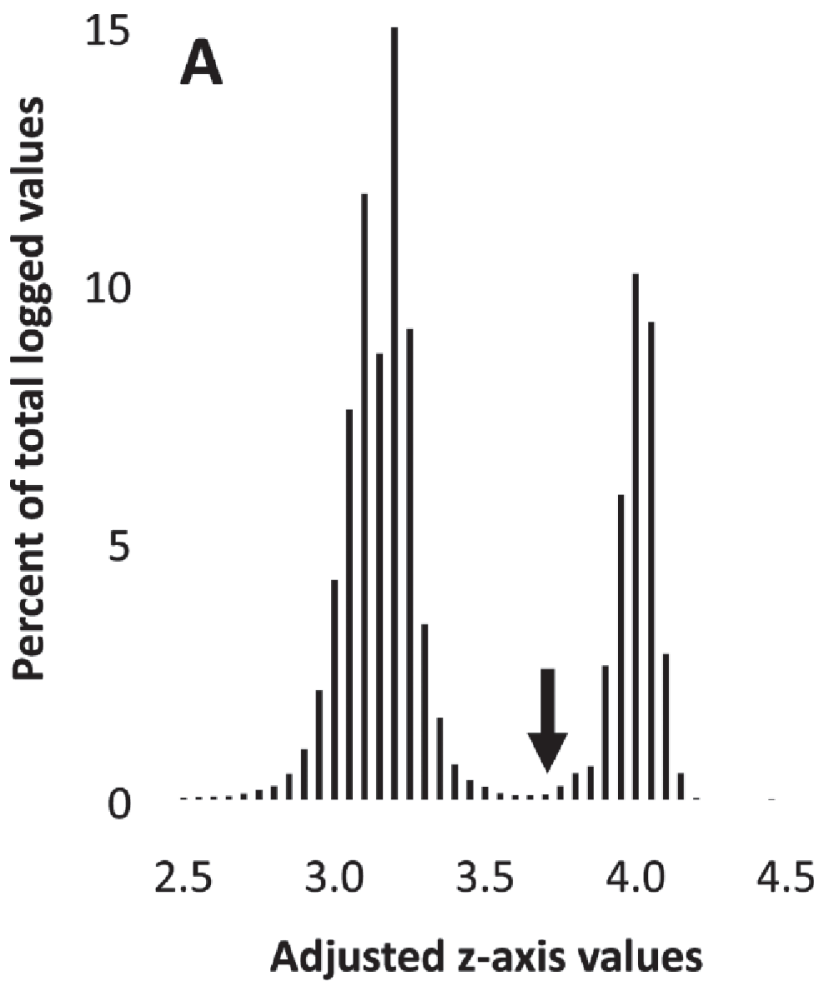

15

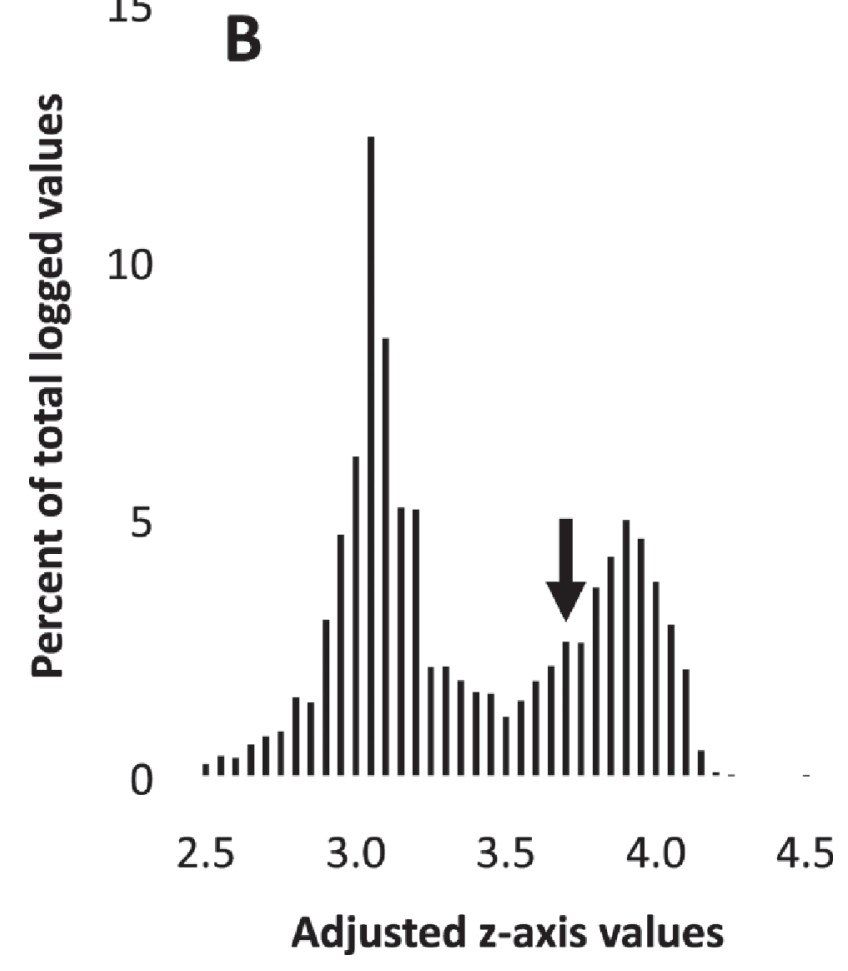

Figure 3. Frequency distributions of the adjusted z-axis data for 2 data loggers over $3 \mathrm{~d}$, plotted in 0.05 bins. Logger A did not rotate around the goat's leg, and has a clear bimodal distribution at the established cut-off point of 3.75 (arrow). Logger B rotated around the goat's leg and lacks a clear bimodal distribution at the established cutoff point of 3.75 (arrow). when interpreting this measure in goats. For instance, the 4 video-monitored does assessed on farm 1 had an average lying bout duration of $62 \pm 20 \mathrm{~min} /$ bout (and $73 \pm 19 \mathrm{~min} /$ bout for the home pen does); these values are less than bout durations reported for cows $(88 \pm$ $30 \mathrm{~min}$; Ito et al., 2009), but still suggestive of goats lying for long periods of time. However, a frequency distribution plot of bout durations (Figure 2) for all farm 1 goats showed a large number of small values; the adult does were very active and had many short lying bouts. Video-monitored does on farm 1 had an average of $15 \pm 5$ lying bouts and $24 \pm 5$ shifts between left and right sides; does from the home pen on this farm had an average of $12 \pm 4$ lying bouts and $21 \pm 5$ shifts between left and right sides. The calculated average of lying bout duration failed to reflect the level of activity shown by the shift between lying sides. This may be due to goats being smaller and lying on a bedded pack, making it possible for them to shift lying orientation without rising. Cows lying on a bedded pack have similar distribution of many, short lying bouts (Ledgerwood et al., 2010). The number of daily lying side changes, and perhaps side changes within a bout, may be a better indicator of restlessness or discomfort.

Data logger application is a challenge. Care is needed to avoid too much tension (resulting in circulation issues) and not enough tension (resulting in logger rotation). On farm 1, where laterality was measured, we noted some data logger rotation around the goats' legs (1 out of 4 loggers rotated in our video-monitored does, and 6 out 30 loggers rotated on the home pen does). Such rotation does not affect lying and standing measures, but prevents laterality calculations, as the z-axis data are unreliable. To ensure that $\mathrm{z}$-axis data has not been confounded by rotation, we strongly recommend that frequency distributions for each logger's z-axis data be plotted and compared against the established cut-off points. Logger data that does not show a clear bimodal distribution (Figure 3) should not be used in laterality calculations.

\section{Objective 3}

Our final objective was to validate the loggers on the younger animals followed in farm 2. The cut-off points established in objective 1 were again used to convert the data into binary values and a 1-min filter was used to remove erroneous data. The PROC FREQ (SAS 9.2) was then used to determine sensitivity, specificity, and percentage of false readings between standing (1) and lying (0) from the video versus the data loggers using the minute-by-minute data for 6 goats. Three days of data were compared, except in the case of 1 goat for which there was only $2 \mathrm{~d}$ of data due to a data log- 
ger battery failure (total data points $=24,480$ ). Data loggers had high sensitivity $(99.8 \%)$ and specificity $(99.4 \%)$ and low false readings (0.36\%). Descriptive statistics of daily lying time and lying bouts were also calculated. The young goats averaged $8.5 \pm 3.2 \mathrm{~h} / \mathrm{d}$ of lying spread over $8 \pm 4$ bouts/d. One goat stood for a very long time each day; when this goat was removed from the calculation, the average lying time was $9.4 \pm$ $1.6 \mathrm{~h} / \mathrm{d}$. Overall, the loggers provided accurate measures of lying time and bouts in younger goats when compared with the video.

\section{Conclusions}

The broad aim of the current study was to validate data loggers for monitoring lying behavior in goats. More specifically, we identified goat-specific cut-off points for $g$-force acceleration data points recorded on the $\mathrm{x}$ - and $\mathrm{z}$-axes and determined the best filter for erroneous data. Using the cut-off points and filter established, we showed that the loggers could record lying behavior in both mature, pregnant does and younger (8-12 mo) goats. Left and right side lying position was established for the mature does; however, caution during application of the loggers was necessary to prevent adversely affecting circulation, while at the same time preventing rotation of the logger around the leg. Overall, we determined that the loggers are appropriate for use in goats to record lying behavior.

\section{ACKNOWLEDGMENTS}

We thank Mark Wynands (University of Guelph) and Bee Li Tan (University of British Columbia), the staff at the Department of Population Medicine, University of Guelph (Guelph, ON), and the staff at Ontario Goat (Guelph, ON). The project was funded by a grant provided by the Ontario Centre of Excellence in Goat Research and Innovation (Peterborough, Ontario, Canada). Gosia Zobel was supported by Canada's Natural Science and Engineering Research Council (Ottawa, ON, Canada) PGSD Scholarship.

\section{REFERENCES}

Andersen, I. L., and K. E. Bøe. 2007. Resting pattern and social interactions in goats - The impact of size and organisation of lying space. Appl. Anim. Behav. Sci. 108:89-103.

Aschwanden, J., L. Gygax, B. Wechsler, and N. M. Keil. 2009. Loose housing of small goat groups: Influence of visual cover and elevated levels on feeding, resting and agonistic behaviour. Appl. Anim. Behav. Sci. 119:171-179.

Bland, J. M., and D. Altman. 1986. Statistical methods for assessing agreement between two methods of clinical measurement. Lancet 1:307-310.

Bonk, S., O. Burfeind, V. S. Suthar, and W. Heuwieser. 2013. Technical note: Evaluation of data loggers for measuring lying behavior in dairy calves. J. Dairy Sci. 96:3265-3271.

Gibbons, J., C. Medrano-Galarza, A. M. de Passillé, and J. Rushen. 2012. Lying laterality and the effect of IceTag data loggers on lying behaviour of dairy cows. Appl. Anim. Behav. Sci. 136:104-107.

Gomez, A., and N. B. Cook. 2010. Time budgets of lactating dairy cattle in commercial freestall herds. J. Dairy Sci. 93:5772-5781.

Ito, K., N. Chapinal, D. M. Weary, and M. A. G. von Keyserlingk. 2014. Associations between herd-level factors and lying behavior of freestall-housed dairy cows. J. Dairy Sci. 97:2081-2089.

Ito, K., M. A. G. von Keyserlingk, S. J. LeBlanc, and D. M. Weary. 2010. Lying behavior as an indicator of lameness in dairy cows. J. Dairy Sci. 93:3553-3560.

Ito, K., D. M. Weary, and M. A. G. von Keyserlingk. 2009. Lying behavior: Assessing within-and between-herd variation in free-stall housed dairy cows. J. Dairy Sci. 92:4412-4420.

Ledgerwood, D. N., C. Winckler, and C. B. Tucker. 2010. Evaluation of data loggers, sampling intervals, and editing techniques for measuring the lying behavior of dairy cattle. J. Dairy Sci. 93:5129-5139

Loretz, C., B. Wechsler, R. Hauser, and P. Rüsch. 2004. A comparison of space requirements of horned and hornless goats at the feed barrier and in the lying area. Appl. Anim. Behav. Sci. 87:275-283. http://dx.doi.org/10.1016/j.applanim.2004.01.005.

Medrano-Galarza, C., J. Gibbons, S. Wagner, A. M. de Passillé, and J. Rushen. 2012. Behavioral changes in dairy cows with mastitis. J. Dairy Sci. 95:6994-7002.

Patt, A., L. Gygax, B. Wechsler, E. Hillmann, R. Palme, and N. M. Keil. 2012. The introduction of individual goats into small established groups has serious negative effects on the introduced goat but not on resident goats. Appl. Anim. Behav. Sci. 138:47-59.

Patt, A., L. Gygax, B. Wechsler, E. Hillmann, R. Palme, and N. M. Keil. 2013. Factors influencing the welfare of goats in small established groups during the separation and reintegration of individuals. Appl. Anim. Behav. Sci. 144:63-72.

Proudfoot, K. L., D. M. Weary, and M. A. G. von Keyserlingk. 2010 Behavior during transition differs for cows diagnosed with claw horn lesions in mid lactation. J. Dairy Sci. 93:3970-3978. 